\title{
Determination of the electroweak couplings of the 3rd generation of quarks at the ILC
}

\author{
A. Irles*, R. Poeschl and F. Richard on behalf of the ILD collaboration \\ Laboratoire de l'Accélerateur Linéaire (LAL), CNRS/IN2P3 et Université de Paris-Sud XI, \\ Centre Scientifique d'Orsay Bâtiment 200, BP 34, F-91898 Orsay CEDEX, France \\ E-mail: irleselal.in2p3.fr
}

This document discuss the prospects on the measurement of the differential cross section as a function of the polar angle of the $e^{-} e^{+} \rightarrow b \bar{b}$ process at $250 \mathrm{GeV}$ centre of mass energy with the International Linear Collider. We show the results for an analysis assuming the integrated luminosity of $2000 \mathrm{fb}^{-1}$ foreseen in the baseline project. The measurement requires determining the charge of both jets identified as originated by a b-quark. The charge measurement is optimally performed using the precise micro-vertex detector of the detector ILD and the charged kaon identification provided by the $d E / d x$ information of its TPC. Thanks to the beam polarisation, we can separate the four independent chirality combinations of the electroweak coupling, enhancing in this way the sensitivity to new physics effects. The prospects on the precision on the electroweak couplings determination are presented and compared with the results from past experiments as LEP or SLC.

European Physical Society Conference on High Energy Physics - EPS-HEP2019 -

10-17 July, 2019

Ghent, Belgium

* Speaker. 


\section{Introduction}

The b-quark electroweak couplings to the Z-boson have been determined [1] by the LEP1 detector collaborations and the SLD Collaboration. LEP experiments extracted the value of $\sin ^{2} \theta_{W}$ from the measurement of several observables as the partial width, $R_{b}$, and the forward-backward asymmetry, $A_{F B}^{b}$. SLD did it from the measurement of the asymmetry in Z interactions with righthanded and left-handed electrons, $A_{L R}$. A discrepancy of 3 standard deviations in $\sin ^{2} \theta_{W}$ was found between the LEP and SLD observations. The most precise of both was the LEP1 measurement, thanks to the higher luminosity acquired $(\sim \times 20$ times more than SLC). Despite the large difference on integrated recorded luminosity, the SLD obtained a similar precision measurement due to the benefits of having a polarised beam and reduced beam spot. Resolving this anomaly is a priority for future $e^{-} e^{+}$colliders.

The International Linear Collider (ILC) $[2,3,4,5,6]$ is a linear electron-positron collider with polarised beams that will produce collisions at several energies. In this document, we will focus on the study of the collisions at centre of mass energy, c.m.e., of $250 \mathrm{GeV}$ (ILC250) but we will also briefly discuss the part of the data taking program foreseen at the Z-pole mass (GigaZ). The International Large Detector (ILD) [6] is one of the proposed detectors to measure the interactions. This detector will be optimised to use Particle Flow (PF) reconstruction algorithms $[7,8,9]$ in order to reconstruct and separate individual particles produced in the collisions. For this, a high granularity calorimetric system is foreseen to be placed inside a $\sim 4 \mathrm{~T}$ magnetic field. Moreover, the ILD will have a high precision tracking system with the first layer placed at $16 \mathrm{~mm}$ of the interaction point to maximise the tracking and vertex reconstruction capabilities. The central tracker of the ILD choice is a Time Projection Chamber (TPC) that provides pattern recognition with more than 200 space points.

\section{Determining the EW couplings from differential cross sections.}

At Born level the differential cross section for bottom-pair production can be written as follows:

$$
\begin{aligned}
& \frac{d \sigma}{d \cos \theta_{b}}\left(e_{L}^{-} e_{R}^{+} \rightarrow b \bar{b}\right) \sim\left(L_{e} L_{b}\right)^{2}\left(1+\cos \theta_{b}\right)^{2}+\left(L_{e} R_{b}\right)^{2}\left(1-\cos \theta_{b}\right)^{2} \\
& \frac{d \sigma}{d \cos \theta_{b}}\left(e_{R}^{-} e_{L}^{+} \rightarrow b \bar{b}\right) \sim\left(R_{e} R_{b}\right)^{2}\left(1+\cos \theta_{b}\right)^{2}+\left(R_{e} L_{b}\right)^{2}\left(1-\cos \theta_{b}\right)^{2}
\end{aligned}
$$

where $\left(L_{e} L_{b}\right)$ etc., are the helicity amplitudes (in this case, left handed associated to the electron and b-quark) that contain the information about the underlying physics i.e. the electroweak couplings to the photon and the $\mathrm{Z}$ or to new bosons. At the ILC, due to the polarised beams, we can probe all four of these amplitudes at a fixed c.m.e.. In first approximation, for back-to-back topologies, the $\cos \theta_{b}$ is defined as the polar angle of the reconstructed momentum $\vec{p}_{b \bar{b}}=\vec{p}_{b}-\vec{p}_{\vec{b}}$.

The helicity amplitudes in Eq. 2.1 have the following detailed form: 


$$
\begin{array}{r}
L_{e} L_{b} \propto \\
Q_{e} Q_{b}+ \\
+\frac{L_{e Z} L_{b Z}}{\sin ^{2} \theta_{W} \cos ^{2} \theta_{W}} B W Z+ \\
+\sum_{Z^{\prime}} \frac{L_{e Z^{\prime}} L_{b Z^{\prime}}}{\sin ^{2} \theta_{W} \cos ^{2} \theta_{W}} B W Z^{\prime}
\end{array}
$$

where $Q_{e}=-1, Q_{b}=-1 / 3$ are the electric charges of the electron and b-quark,respectively. $L_{e Z}=$ $I_{e 3}-Q_{e} \sin ^{2} \theta_{W}, L_{b Z}=I_{b 3}-Q_{b} \sin ^{2} \theta_{W}$, are the general form of the electroweak coupling to the $\mathrm{Z}$. Left-handed is defined by $I_{3}=1 / 2$ and right handed by $I_{3}=0 . B W Z=s /\left(s-M_{z}^{2}\right)$ is the Breit Wigner of the Z-boson. Similarly is for the $L_{e Z^{\prime}}$ and $L_{b Z^{\prime}}$ but for new predicted resonances, $Z^{\prime}$, resulting from a similar symmetry than the $\mathrm{SM}$ (i.e. $\left.S U(2)_{R}\right)$. This formula can be written for all possible combinations $L_{e} L_{b}, L_{e} R_{b}, R_{e} R_{b}, R_{e} L_{b}$

The determination of $L_{e} L_{b}$ with high precision will be possible at the ILC running at $250 \mathrm{GeV}$. We can safely assume that the $Q_{e} Q_{b}$ part, the electric charges, would be known due to $U(1)_{e m}$ gauge invariance and that the standard model Z-boson couplings to the electron and b-quark (Eq line. 2.2c) would be well measured at ILC GigaZ at the subpercent level of precision. Therefore, ILC250 will be able to quantify contributions from new particles (Eq line. 2.2d). Beam polarisation enables determining the chiral structure of the couplings to the new boson. Many theoretical models predicte deviations for the right handed couplings that as of today are only weakly constrained by experiment. Prospects on this will be given in Section 4.

A convenient rearrangement of the helicity terms in Eqs. 2.1 will be also used in the following:

$$
f_{\text {pol }}(S, A)=S_{\text {pol }}\left(1+\cos ^{2} \theta_{b}\right)+A_{\text {pol }} \cos \theta_{b}
$$

where $f_{p o l}$ stands for the $e^{-} e^{+}$cross section with a polarised beams. The $S$ term is related to the inclusive cross section while $A$ is simply related with the $A_{F B}^{b}$ without requiring a significant correction.

\section{Event reconstruction and selection}

All results shown here are obtained using full simulation of the ILD concept. The size of the analysed samples is the equivalent of $250 \mathrm{fb}^{-1}$ for each of the processes. The experimental studies are made for $100 \%$ beam polarisation. Final results will be scaled to the realistic beam polarisation $P\left(e^{-}, e^{+}\right)=( \pm 0.8, \mp 0.3)$ The events are generated at leading order using the WHIZARD 1.95 [10, 11] event generator. The hadronisation, and final state radiation is simulated by the Pythia 6.422 event generator [12] which also models the $B^{0}-\overline{B^{0}}$ oscillations. The ILD detector geometry and the interaction of the particles with the detector are simulated within the Mokka framework interfaced with the Geant4 toolkit $[13,14,15]$. The different reconstruction algorithms are implemented in the ILCSoft toolkit. We make use of the tracking, b-tagging, particle identification in the TPC and jet clustering algorithms described in Ref.[16]. The primary and secondary vertex reconstruction has special importance for this analysis. Therefore they were optimised to fit the high precision requirements of this analysis (see, again, Ref. [16] for more details). 
The event pre-selection proceeds as follows: we reconstruct events with two jets using the Durham algorithm. Events that are subject to a radiative return to the Z-pole are rejected by a combination of two cuts: we require that that the two jet system has invariant mass larger than $180 \mathrm{GeV}$ and/or that if a photon is reconstructed inside of the detector, it should an have energy lower than $40 \mathrm{GeV}$ to remove events with a visible ISR photon. Further, a cut in the sum of the two jet masses is applied: if the sum of the two jet masses is greater than $120 \mathrm{GeV}$, the event is rejected. This cut helps reducing the impact of QCD final state radiation that dilutes the backto-back configuration of the two jets and also helps suppressing the remaining background from $\mathrm{ZZ}$ events. For the final step of our pre-selection of $e^{-} e^{+} \rightarrow b \bar{b}$, we suppress the most dominant background, the $e^{-} e^{+} \rightarrow q \bar{q}(q=u, d, s, c)$, by requiring that one of the reconstructed jets has a large $b$-tag value (b-tag $>0.9)$ and the other has at least $b$-tag $>0.2$. These cuts allow for a consistent selection of a $b \bar{b}$ dijet system with invariant masses between 200 and $250 \mathrm{GeV}$. The impact of these cuts on the signal selection efficiency and the ratio of background vs signal is summarised in Table 1. This selection corresponds to a $10 \%$ correction with respect to the Born cross section. For the angular dependence, this correction is much smaller (negligible for $e_{L}^{-} e_{R}^{+}$and $\sim 1 \%$ for $e_{R}^{-} e_{L}^{+}$).

\subsection{Charge measurement}

After the selection of a highly pure $b \bar{b}$ sample, we need to determine the jet charges to reconstruct the angular distribution. The $\mathrm{b}$ quarks hadronise in $40 \%$ percent of the cases in $B^{ \pm}$-mesons, $40 \%$ of the cases in neutral B-mesons, $10 \%$ of the cases in strange B-mesons and $10 \%$ of the cases into B-baryons. B-hadrons have lifetime of about $c \tau=400-500 \mu \mathrm{m}$ giving rise to well measurable secondary vertices. Around $80 \%$ of the B-mesons yield charged Kaons in the final state. The Bmesons will then decay in $\mathrm{D}^{0}$ or $\mathrm{D}^{ \pm}$mesons in about the $80 \%$ of the cases. We can infer the charge of the originating b-quark by measuring the charge of the hadrons created in the b-quark hadronisation process. Therefore we can apply two methods to measure the charge of the b-quark. The first method uses the charge of the secondary vertex based on micro-vertex tracking (Vtx-method) and the second uses charged kaons identified in the TPC (K-method). Both methods rely on the efficient and full reconstruction of secondary and tertiary vertices. While ILD has a $99 \%$ probability to reconstruct the relevant charged tracks, this probability falls to $96 \%$ for tracks connected to the micro-vertex and having a significant offset. Revisiting the tracking and vertexing algorithms to improve this value is an ongoing activity in the collaboration. This probability drops rapidly for jets reconstructed at $\left|\cos \theta_{b}\right|>0.9$ due to imprecise track reconstruction outside of the acceptance of the micro-vertex detector. Modifying this geometry and/or prolonging the first layer of the barrel is under consideration by the collaboration. Due to the high granularity of the ILD TPC, the power of separation of kaons and other hadrons is large enough to provide kaon identification with high efficiency and purity for jets with $\left|\cos \theta_{b}\right|<0.9$ and momentum above $3 \mathrm{GeV}$. For this process, kaons are identified with a purity reaching $90 \%$ at $88 \%$ efficiency.

The charge measurement allows us to attribute a $\operatorname{sign}$ to the reconstructed $\cos \theta_{b}$ value. However a sign-flip may be induced due the possibility of $B^{0}-\overline{B^{0}}$ oscillations, to missed tracks in a reconstructed vertex and the misidentification of kaons. In order to correct for that, we apply a double charge measurement in which we only keep events in which both jets have compatible charges. Even in this case, it exists the possibility that both charges are wrongly estimated and the total sign of the $\cos \theta_{b}$ is inverted. To correct for this effect, we estimate the fraction of mistakes 

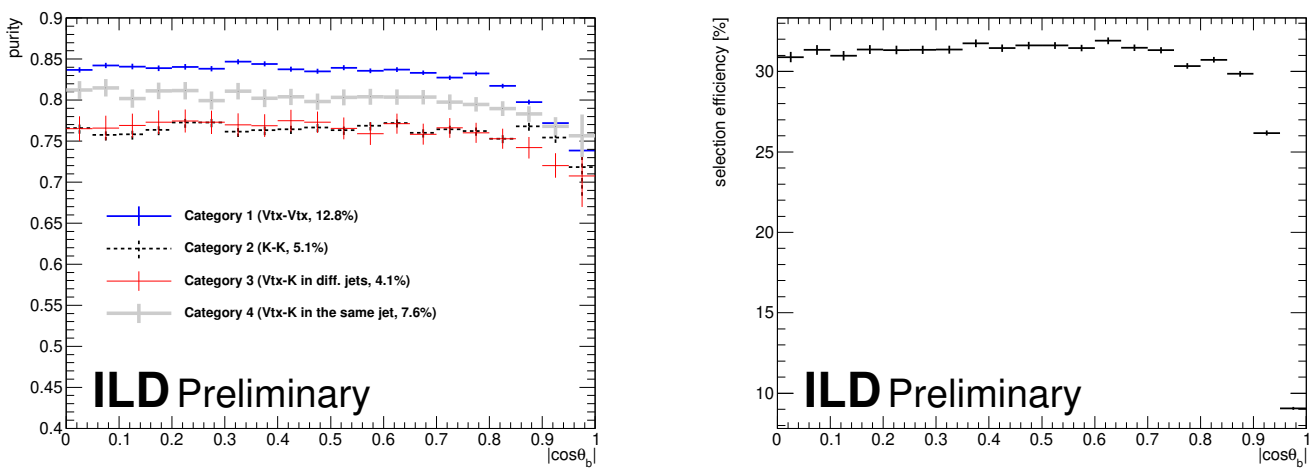

Figure 1: Left: measurement of the purity or probability of a correct assignment of the charge for the different categories defined in the text. The numbers given in parenthesis correspond to the contribution of each category to the final efficiency of selection of each category. The selection is made in a sequential way: if an event is not in the first category, then we check the second and so forth. Right: Final efficiency of selection as a function of $\cos \theta_{b}$

by using the events with the two jets with incompatible charge measurements. We separate our sample $\left(N_{\text {sample }}\right)$ in two subsets: the accepted events with compatible charges in both jets $\left(N_{a}\right)$ and the rejected events with same charge measured in both jets $\left(N_{r}\right)$ :

$$
\begin{aligned}
N_{a}\left(\cos \theta_{b}\right) & =p^{2}\left(\left|\cos \theta_{b}\right|\right) \cdot N_{\text {sample }}\left(\cos \theta_{b}\right)+q^{2}\left(\left|\cos \theta_{b}\right|\right) \cdot N_{\text {sample }}\left(-\cos \theta_{b}\right) \\
N_{r}\left(\left|\cos \theta_{b}\right|\right) & =2 \cdot p\left(\left|\cos \theta_{b}\right|\right) \cdot q\left(\left|\cos \theta_{b}\right|\right) \cdot N_{\text {sample }}\left(\left|\cos \theta_{b}\right|\right)
\end{aligned}
$$

where $p$ is the probability of a correct assignment of the charge, purity, and $q=1-p$. The purity value can be extracted by solving the Eq. 3.2 and then can be used to correct for migrations. Moreover, since we have two different methods to determine the charge, we can perform the same analysis for different combinations of the method to be used. The different combinations are classified in four categories: the charge of both jets measured by using the Vtx-method; same but using the K-method; one jet charge measured with the Vtx-method and the other with the K-method; and, finally, only one jet with charge measurement but with the two methods. The measured purities for the different categories is shown in the left plot of Figure 1. The recovery procedure gives an approximately constant $p\left(\cos \theta_{b}\right)$ except for the very forward region $\left|\cos \theta_{b}\right|>0.9$ where there is also a significant drop of efficiency. The total efficiency of the selection after applying the double charge measurements is shown in the last row of Table 1 and in the right part of Figure 1. We see that the efficiency is mostly homogeneous in the barrel region and drops sharply in the very forward region due to lost tracks or partially reconstructed vertices.

\section{Results}

The angular distributions for the two polarisations after correcting for migrations and detector inhomogeneities (see Figure 1 for both) are shown in Figure 2. The function described in Eq. 2.3 


\begin{tabular}{|c|c|c|c|c|c|c|c|c|}
\hline \multicolumn{9}{|c|}{$e_{L}^{\bar{e}} e_{R}^{+} \rightarrow b \bar{b}$} \\
\hline & Signal [\%] & \multicolumn{7}{|c|}{ Background processes $[\%]$ (B/S [\%]) } \\
\hline & $b \bar{b}$ & $c \bar{c}$ & $q \bar{q}(u d c s)$ & $\gamma b \bar{b}$ & ZZ & WW & $\mathrm{HZ}$ & Total \\
\hline Pre-selection & 65.9 & $0.6(1.2)$ & $0.1(0.1)$ & $0.1(0.6)$ & $5.6(2.0)$ & $0.0(0.1)$ & $10.7(0.3)$ & $0.3(4.3)$ \\
\hline Double charge & 29.2 & $0.0(0.0)$ & $0.1(0.5)$ & $0.0(0.5)$ & $0.6(0.5)$ & $0.0(0.0)$ & $4.9(0.3)$ & $0.1(2.1)$ \\
\hline \multicolumn{9}{|c|}{$e_{R}^{-} e_{L}^{+} \rightarrow b \bar{b}$} \\
\hline & Signal [\%] & \multicolumn{7}{|c|}{ Background processes $[\%](\mathrm{B} / \mathrm{S}[\%])$} \\
\hline & $b \bar{b}$ & $c \bar{c}$ & $q \bar{q}(u d c s)$ & $\gamma b \bar{b}$ & $\mathrm{ZZ}$ & WW & $\mathrm{HZ}$ & Total \\
\hline Pre-selection & 65.6 & $0.6(2.4)$ & $0.0(0.1)$ & $0.1(0.9)$ & $5.8(0.9)$ & $0.0(0.0)$ & $10.7(1.5)$ & $0.2(5.7)$ \\
\hline Double charge & 29.1 & $0.1(1.4)$ & $0.0(0.0)$ & $0.0(0.7)$ & $2.3(0.9)$ & $0.0(0.0)$ & $4.7(1.5)$ & $0.1(4.5)$ \\
\hline
\end{tabular}

Table 1: Percentage of the signal and the different background events remaining after each selection step. For completeness, the background to signal ratio (in \% units) is also quoted.
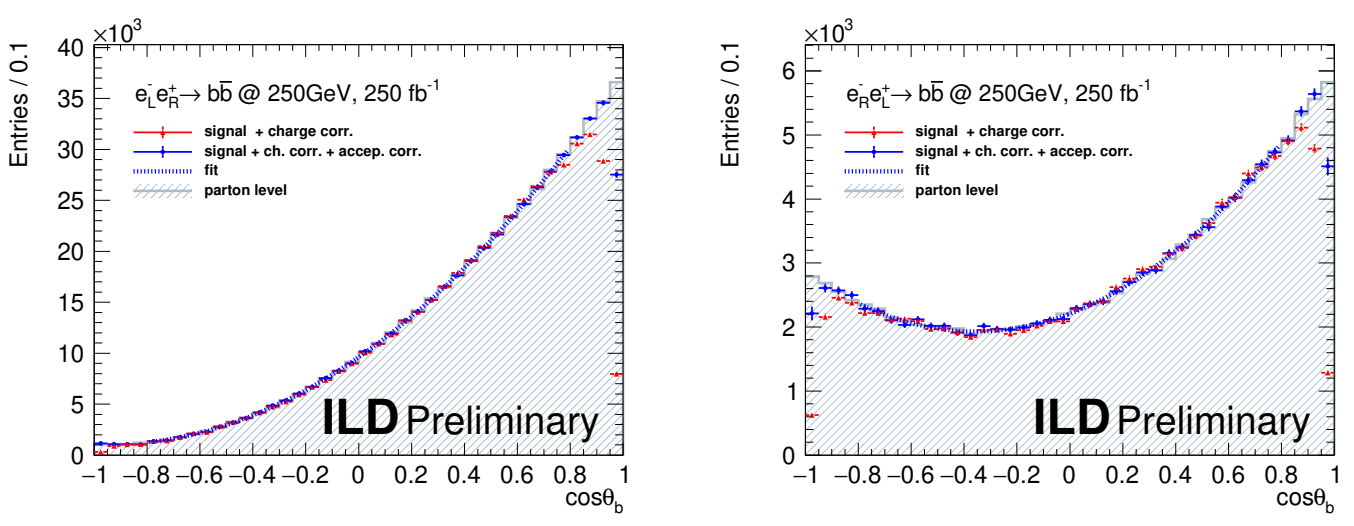

Figure 2: Results of the fit described in Eq. 2.3 for pure left and pure right polarisations after background subtraction and all corrections.

is fitted to both distributions restricted to $\left|\cos \theta_{b}\right|<0.8$ to avoid the regions with large drops of efficiency. In both cases we see the perfect agreement between reconstructed distribution at detector level and at parton level. The results from the fit can be extrapolated to match the realistic scenario of ILC running at $250 \mathrm{GeV}$ in which $2000 \mathrm{fb}^{-1}$ of statistics for different beam polarisations will be collected. Table 4 summarises the results on the expected precisions. It shows the estimations of statistical and systematic uncertainties. The main systematic uncertainties affecting the measurements are the background subtraction, the errors due to beam polarisation estimation and the b-tagging efficiency. We assume that the background will be known at the $\sim 1 \%$ level and that the errors in the polarisation will be as the ones described in reference [4]. For the b-tagging efficiency measurements we can follow a similar strategy as in Ref. [17]. Current studies show that the uncertainty on this efficiency can be measured at the $0.1 \%$ precision, including the possible effects of jet correlations which are largely suppressed due to the small beam spot foreseen for ILC. A full study and description of the systematic uncertainties estimation is left for a future publication. From this table, the precision on the determination of the electroweak couplings can be derived. Furthermore, these reconstructed distributions can also be interpreted assuming the presence of new resonances as described in Eq. 2.2a. 


\begin{tabular}{c|c|c}
\hline & \multicolumn{2}{|c}{ Beam Polarisations $\left(e^{-}, e^{+}\right)$} \\
& $\mathbf{( - 8 0 , 3 0 )}$ & $\mathbf{( 8 0 , - 3 0 )}$ \\
\hline Selection Eff. [\%] & 29 & 29 \\
Luminosity [ $\mathrm{fb}^{-1}$ ] & 900 & 900 \\
$\Delta S$ (syst.+stat.) [\%] & $0.095+0.15$ & $0.20+0.17$ \\
$\Delta A$ (syst.+stat.) [\%] & $0.13+0.15$ & $0.77+0.26$ \\
\hline
\end{tabular}

Table 2: Estimation of the achievable precision on $S$ and $A$ described in Eq. 2.3
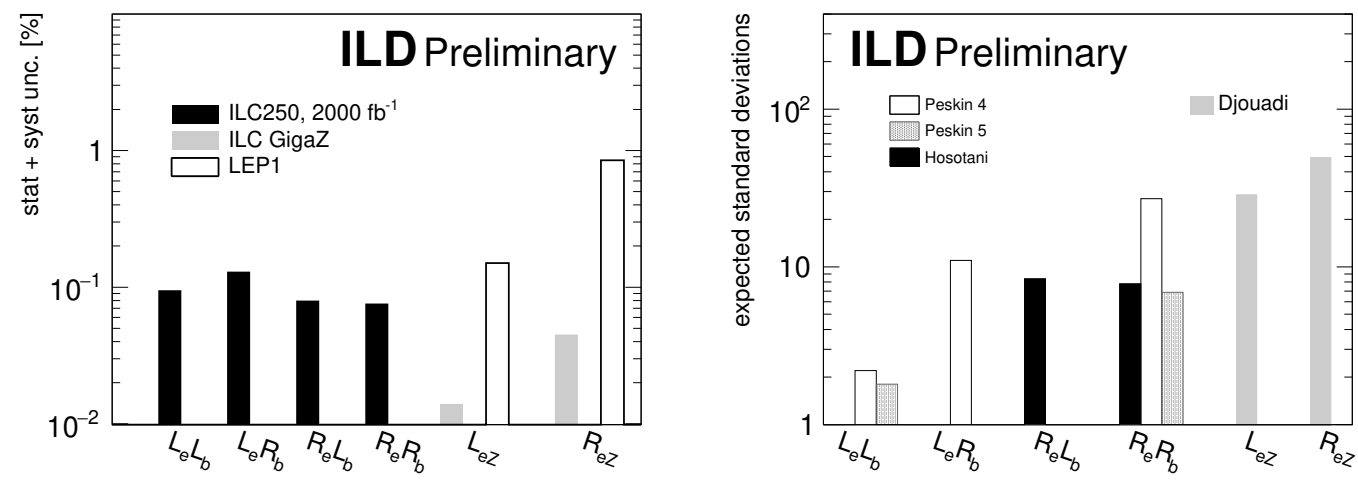

Figure 3: Left: Expected precision on the b-quark electroweak couplings. Right: Expected number of standard deviations for $3 \mathrm{RS}$ models. For Djouadi [18] one assumes $m_{Z^{\prime}}=3 \mathrm{TeV}$. For the Peskin et al. model [19], two versions are given, labelled as Peskin 4 and Peskin 5. For Hosotani et al.[20] one assumes $m_{Z^{\prime}} \sim 8 \mathrm{TeV}$ for the 3 resonances.

The results on the expected precisions foreseen for ILC compared with LEP1 are summarised in the left part of Figure 3. The level of accuracy foreseen would allow resolving unambiguously the LEP1 anomaly. The accuracy opens sensitivity of the helicity amplitudes to propagators from new bosons as they appear e.g. in Randall-Sundrum models. The right part of Fig. 3 shows that masses of new Z' bosons $[18,19,20]$ that can be probed at ILC250.

\section{Conclusions and prospects}

This document summarises the results of a realistic analysis based on full detector simulation and reconstruction of $e^{-} e^{+} \rightarrow b \bar{b}$ processes at the ILC. The results show a large improvement on the reachable precisions compared with previous experiments allowing for an unambiguously resolution of the SLC/LEP1 anomaly in the $\sin ^{2} \theta_{W}$, determination. Also discussed is a major advantage compared with other experiments with no beam polarisation: the power of separation and the independent determination of the left and right handed components of the electroweak couplings. Several technical aspects of the analysis presented here are not discussed in detail but will be covered in a future publication signed by the ILD collaboration.

In addition, similar studies but on $e^{-} e^{+} \rightarrow t \bar{t}$ are being also conducted within the ILD collaboration and will be a subject of another publication. 


\section{Acknowledgements}

We thank the ILD group for event generation and simulation software development works. The research leading to these results has received funding from the People Programme (Marie Curie Actions) of the European Unions Seventh Framework Programme (FP7/2007-2013) under REA grant agreement, PCOFUND-GA-2013-609102, through the PRESTIGE programme coordinated by Campus France.

\section{References}

[1] ALEPH, DELPHI, L3, OPAL, SLD, LEP ElECTROWEAK Working GRoup, SLD Electroweak Group, SLD HeAVy FlaVour Group collaboration, Precision electroweak measurements on the Z resonance, Phys. Rept. 427 (2006) 257 [hep-ex/ 0509008 ].

[2] T. Behnke, J. E. Brau, B. Foster, J. Fuster, M. Harrison, J. M. Paterson et al., The International Linear Collider Technical Design Report - Volume 1: Executive Summary. , 1306.6327.

[3] H. Baer, T. Barklow, K. Fujii, Y. Gao, A. Hoang, S. Kanemura et al., The International Linear Collider Technical Design Report - Volume 2: Physics. , 1306.6352.

[4] C. Adolphsen, M. Barone, B. Barish, K. Buesser, P. Burrows, J. Carwardine et al., The International Linear Collider Technical Design Report - Volume 3.I: Accelerator \& in the Technical Design Phase. , 1306.6353.

[5] C. Adolphsen, M. Barone, B. Barish, K. Buesser, P. Burrows, J. Carwardine et al., The International Linear Collider Technical Design Report - Volume 3.II: Accelerator Baseline Design. , 1306.6328.

[6] H. Abramowicz et al., The International Linear Collider Technical Design Report - Volume 4: Detectors, 1306.6329.

[7] J.-C. Brient and H. Videau, The Calorimetry at the future $e+e$ - linear collider, eConf $\mathbf{C 0 1 0 6 3 0}$ (2001) E3047 [hep-ex/0202004].

[8] V. Morgunov and A. Raspereza, Novel 3-D clustering algorithm and two particle separation with tile HCAL, physics/0412108.

[9] Sefkow, F. and White, A. and Kawagoe, K. and Pöschl, R. and Repond, J., Experimental Tests of Particle Flow Calorimetry, Rev. Mod. Phys. 88 (2016) 015003 [1507. 05893 ].

[10] W. Kilian, T. Ohl and J. Reuter, WHIZARD: Simulating Multi-Particle Processes at LHC and ILC, Eur. Phys. J. C71 (2011) 1742 [0708.4233].

[11] M. Moretti, T. Ohl and J. Reuter, O'Mega: An Optimizing matrix element generator, hep-ph/0102195.

[12] T. Sjöstrand, S. Mrenna and P. Skands, PYTHIA 6.4 physics and manual, Journal of High Energy Physics 2006 (2006) 026.

[13] GEANT4 collaboration, GEANT4: A Simulation toolkit, Nucl. Instrum. Meth. A506 (2003) 250.

[14] J. Allison et al., Geant4 developments and applications, IEEE Trans. Nucl. Sci. 53 (2006) 270.

[15] J. Allison et al., Recent developments in Geant4, Nucl. Instrum. Meth. A835 (2016) 186.

[16] S. Bilokin, Hadronic showers in a highly granular silicon-tungsten calorimeter and production of bottom and top quarks at the ILC, theses, Paris Saclay, July, 2017. 
[17] DELPHI collaboration, A Precise measurement of the partial decay width ratio $R(b) * * 0=$ Gamma(b anti-b) / Gamma(had), Eur. Phys. J. C10 (1999) 415.

[18] A. Djouadi, G. Moreau and F. Richard, Resolving the $A(F B)^{* *} b$ puzzle in an extra dimensional model with an extended gauge structure, Nucl. Phys. B773 (2007) 43 [hep-ph / 0610173$].$

[19] J. Yoon and M. E. Peskin, Fermion Pair Production in SO(5) x U(1) Gauge-Higgs Unification Models, 1811.07877.

[20] S. Funatsu, H. Hatanaka, Y. Hosotani and Y. Orikasa, Distinct signals of the gauge-Higgs unification in $e^{+} e^{-}$collider experiments, Phys. Lett. B775 (2017) 297 [1705. 05282]. 